\title{
Impact of a bladder cuff excision during radical nephroureterectomy on cancer specific survival in patients with upper tract urothelial cancer in Korea: a retrospective, multi-institutional study
}

\author{
Yun-Sok HA ${ }^{1}$, Jae-Wook CHUNG ${ }^{1}$, Seock H. CHOI ${ }^{1}$, Jun N. LEE ${ }^{1}$, Bum S. KIM ${ }^{1}$, \\ Tae-Hwan KIM ${ }^{1}$, Eun S. YOO ${ }^{1}$, Tae G. KWON ${ }^{1}$, Seok-Soo BYUN ${ }^{2}$, Young D. CHOI ${ }^{3}$, \\ Ho W. KANG ${ }^{4}$, Seok J. YUN ${ }^{4}$, Wun-Jae KIM ${ }^{4}$, Hyun T. KIM ${ }^{1}$ *
}

${ }^{1}$ Department of Urology, Kyungpook National University School of Medicine, Daegu, South Korea; ${ }^{2}$ Department of Urology, Seoul National University Bundang Hospital, Seongnam, South Korea; ${ }^{3}$ Department of Urology, Severance Hospital, Urological Science Institute, Yonsei University College of Medicine, Seoul, South Korea; ${ }^{4}$ Department of Urology, Chungbuk National University Hospital, Chungbuk National University College of Medicine, Cheongju, South Korea

*Corresponding author: Hyun T. Kim, Department of Urology, School of Medicine, Kyungpook National University, 130 Dongdeokro, Jung-gu, Daegu 41944, South Korea. E-mail: urologistk@knu.ac.kr

\section{A B S T R A C T}

BACKGROUND: Radical nephroureterectomy (RNU) with bladder cuff excision (BCE) is the surgical principle adopted for the treatment of upper tract urothelial cancers (UTUCs). However, not all RNUs are performed with BCE. We quantified the prognostic impact of RNU with BCE on cancer-specific survival (CSS) in a large patient population.

METHODS: In total, 505 patients with UTUC were enrolled from four different institutions. The clinicopathological parameters of patients who underwent RNU with and without BCE were compared. The Kaplan-Meier and multivariate Cox regression analyses were performed to assess the influence of BCE on CSS.

RESULTS: In total, 60 (11.9\%) patients had not undergone BCE during RNU. Compared to patients who underwent $\mathrm{BCE}$, these patients were older and had more comorbidities. Patients with UTUC who had not undergone BCE were more likely to be associated with $\geq \mathrm{pT} 3$, margin positivity, and renal pelvis localization compared to patients who underwent BCE. Median follow-up periods were 30.5 months (range, 6-144 months). The Kaplan-Meier estimates revealed that BCE during RNU was not significantly associated with CSS in all UTUC patients and in the subgroup with renal pelvis localization; however, patients who underwent RNU without BCE had significantly worse CSS rates compared to patients who underwent RNU with BCE in the subgroup analysis of patients with ureteral cancer. Multivariate analysis identified $\mathrm{BCE}$ as an independent prognostic factor of CSS in patients with ureteral cancer.

CONCLUSIONS: In the present study, RNU without BCE resulted in significantly worse CSS in ureteral cancer patients, which indicated that BCE should be mandatory in patients with ureteral cancer.

(Cite this article as: Ha YS, Chung JW, Choi SH, Lee JN, Kim BS, Kim TH, et al. Impact of a bladder cuff excision during radical nephroureterectomy on cancer specific survival in patients with upper tract urothelial cancer in Korea: a retrospective, multi-institutional study. Minerva Urol Nefrol 2017;69:466-74. DOI: 10.23736/S0393-2249.17.02807-7)

Key word: Nephrectomy - Urinary bladder neoplasms - Surgical procedure, operative.

$\mathrm{U}$ pper tract urothelial carcinoma (UTUC) is a rare disease that accounts for approximately $5 \%$ of all tumors derived from the uro- thelium with an annual incidence of 1-2 cases per 100,000 people but it is a potentially lethal disease. ${ }^{1-3}$ Despite intense collaborative efforts 
to improve the knowledge on this disease, its management remains challenging. ${ }^{4}, 5$ In patients with a normal contralateral kidney, radical nephroureterectomy (RNU) with bladder cuff excision (BCE) is the gold standard treatment for UTUC.6,7 However, not all RNUs are performed with a BCE. According to a previous multi-institutional tertiary care center study, a substantial portion of the patients who underwent RNU (711/1249, 56.9\%) did not undergo BCE. ${ }^{8}$ Moreover, urologists have disputed whether they should always perform BCE during RNU since the introduction of minimally invasive surgery. ${ }^{9}$ Tumor recurrence in a retained ureteral segment after imperfect RNU is an important dilemma and may contribute to bladder recurrence or metastasis. ${ }^{10}$ Oncologic outcome is a considerable issue for patients who undergo RNU with or without BCE. Many studies have shown poor oncologic outcomes in patients who underwent RNU without BCE, whereas some studies have reported comparable outcomes between the two surgical approaches. . $^{8}, 11-13$

In some cases, we had not performed BCE during RNU owing to several inevitable reasons. For example, surgeons omitted BCE in elderly patients or in patients having multiple comorbidities, for whom the operation had to be completed in a short time. In cases of patients who had aggressive tumors in the involved ureters with distal parts highly adhesive to the adjacent organs, surgeons failed to approach the end of the ipsilateral ureters near the bladder due to technical challenges and therefore they omitted BCE. To date, there have not been any definitive conclusions concerning the prognostic value of $\mathrm{BCE}$ in the Korean UTUC patient cohort. Therefore, we compared the clinical characteristics and cancer-specific survival (CSS) between UTUC patients who underwent RNU with and without BCE in a large, multi-institutional study.

\section{Materials and methods}

\section{Ethics statement}

This study was approved by the institutional review board at Kyungpook National Univer- sity, Daegu, South Korea (KNUMC 2016-05021). The study was carried out in agreement with the applicable laws and regulations, good clinical practices, and ethical principles as described in the Declaration of Helsinki. The board exempted informed consent because it was a retrospective study.

\section{Study population}

A database of 505 patients with UTUC who underwent either open or laparoscopic RNU between 2001 and 2013 at four academic centers was reviewed. The patient characteristics included in the database were the age, sex, Body Mass Index, history of bladder cancer, pre-operative American Society of Anesthesiologists physical status score, surgical approach (open vs. laparoscopic), tumor pathology (stage, grade, lymph node status, and lymphatic vessel invasion), tumor necrosis, concomitant carcinoma in situ, tumor location, use of adjuvant chemotherapy, unifocal or multifocal disease, disease recurrence or metastasis, and mortality from urothelial carcinoma. The complete follow-up data were available for all patients and were therefore included in the analysis. After combining the datasets, reports were generated for each variable to identify the inconsistencies in the data along with other data integrity problems. Before the final analysis, the database was frozen, and the final dataset was generated. In order to avoid bias in the survival estimates, patients who had previous or concomitant muscle-invasive bladder tumors treated by cystectomy were excluded. Patients with distant metastases at diagnosis and those who received neoadjuvant therapy were also excluded. All patients underwent preoperative cystoscopy, urine cytology, and chest and abdominal-pelvic computed tomography.

\section{Surgical techniques}

Surgery was performed according to the standard RNU protocol that includes the extrafascial dissection of the kidney along with the entire length of the ureter and the adja- 


\section{COPYRIGHT ${ }^{\odot} 2017$ EDIZIONI MINERVA MEDICA}

cent segment of the bladder cuff. BCE was performed according to the standard protocol (i.e., using an extravesical approach involving a Gibson incision) as stipulated by each center. The hilar and regional lymph nodes adjacent to the ipsilateral great vessel were generally resected if they were palpable intraoperatively or if they were enlarged on preoperative axial imaging. The extent of lymphadenectomy performed was at the discretion of the individual surgeons. Majority of the patients with non-organ-confined diseases received cisplatin-based adjuvant chemotherapy. Tumors were staged according to the $6^{\text {th }}$ edition of the American Joint Committee on Cancer staging system, ${ }^{14}$ and the tumor grades were assessed according to the 1998 World Health Organization classification system. ${ }^{15}$

\section{Follow-up}

Each patient was followed up according to the standard protocol. In general, patients were followed up every 3-4 months during the first year following RNU, every 6 months in years 2-5, and annually thereafter. The follow-up consisted of taking the patient history, physical examination, routine blood and serum chemistry tests, urinary cytology, chest radiography, cystoscopic evaluation of the urinary bladder, and radiographic evaluation of the contralateral upper urinary tract. Elective bone scans, chest computed tomography scans, and magnetic resonance imaging were performed when clinically indicated. Time to CSS was calculated as the time from surgery to the date of cancer-related mortality.

\section{Statistical analysis}

Categorical variables were compared using the Pearson $\chi^{2}$ or Fisher exact tests and continuous variables were compared using the Student $t$-test. The probability of survival was calculated using the Kaplan-Meier method, and statistical differences were evaluated using the log-rank test. Multivariate analysis was performed using the Cox proportional hazards model adjusted for the effect of potential confounders. All statistical analyses were performed using the SPSS software version 18.0 (SPSS Inc., Chicago, IL, USA). All reported $\mathrm{P}$ values were two-sided, and all $\mathrm{P}<0.05$ were considered statistically significant.

\section{Results}

The study population consisted of 505 patients with UTUC who underwent RNU with $(445 / 505,88.1 \%)$ and without $(60 / 505,11.9 \%)$ BCE. Compared to patients who underwent $\mathrm{BCE}$, patients who did not undergo $\mathrm{BCE}$ were older $(\mathrm{P}<0.001)$ and had a higher American Society of Anesthesiologists performance status score $(\mathrm{P}=0.009$; Table I). UTUC treated without BCE was more likely to be associated with pathologic stage $\geq \mathrm{T} 3$ than UTUC treated with $\mathrm{BCE}(\mathrm{P}=0.026)$. Patients who did not undergo $\mathrm{BCE}$ had higher potential of surgical margin positivity than that of patients who underwent $\mathrm{BCE}(\mathrm{P}=0.001)$. Compared to the tumors of patients treated with BCE, those of patients treated without $\mathrm{BCE}$ were more frequently localized to the renal pelvis $(\mathrm{P}=0.001)$. Other clinicopathological features were similar between the two groups (Table I).

During the follow-up (median, 30.5 months; range, 6-144 months), 173 patients (34.3\%) experienced intravesical recurrence and 137 patients $(27.1 \%)$ developed locoregional recurrence/distant metastasis. There were no significant differences in the intravesical recurrencefree survival and local/distant metastasis-free survival between patients who underwent and did not undergo BCE even in subgroup analyses of renal pelvic tumor and ureteral cancer. In total, 111 patients $(22.0 \%)$ died during the follow-up. Of the 111 patients who died, the deaths of 88 patients $(17.4 \%)$ were UTUC related. The Kaplan-Meier estimates for CSS are demonstrated in Figure 1. The log-rank analysis revealed that BCE during RNU was not significantly associated with CSS in all UTUC patients and in the subgroup of renal pelvis cancer; however, Kaplan-Meier analysis revealed that patients who underwent RNU without BCE had significantly worse CSS rates than patients who underwent RNU with 


\section{COPYRIGHT ${ }^{\odot} 2017$ EDIZIONI MINERVA MEDICA}

BLADDER CUFF EXCISION DURING RADICAL NEPHROURETERECTOMY

HA

TABLE I.—Clinicopathological characteristics of patients with and without bladder cuff excision.

\begin{tabular}{|c|c|c|c|}
\hline Parameters & $\begin{array}{l}\text { Patients without bladder } \\
\text { cuff excision }(\mathrm{N} .=60)\end{array}$ & $\begin{array}{l}\text { Patients with bladder } \\
\text { cuff excision }(\mathrm{N} .=445)\end{array}$ & $\mathrm{P}$ \\
\hline Age (years, mean \pm SD) & $72.0 \pm 9.6$ & $65.5 \pm 10.4$ & $<0.001$ \\
\hline BMI $\left(\mathrm{kg} / \mathrm{m}^{2}\right)$ & $23.4 \pm 2.7$ & $23.8 \pm 3.1$ & 0.365 \\
\hline Sex, N. (\%) & & & 0.137 \\
\hline Men & $36(60.0)$ & $312(70.1)$ & \\
\hline Women & $24(40.0)$ & $133(29.9)$ & \\
\hline Smoking status, N. (\%) & & & 0.152 \\
\hline No & $44(73.3)$ & $283(63.6)$ & \\
\hline Yes & $16(26.7)$ & $162(36.4)$ & \\
\hline ASA score, N. (\%) & & & 0.009 \\
\hline 1 & $6(10.0)$ & $114(25.6)$ & \\
\hline $2-4$ & $54(90.0)$ & $331(74.4)$ & \\
\hline Laterality, N. (\%) & & & 0.584 \\
\hline Left & $34(56.7)$ & $234(52.6)$ & \\
\hline Right & $26(43.3)$ & $211(47.4)$ & \\
\hline Operative method & & & 0.319 \\
\hline Open & $18(30.0)$ & $165(37.1)$ & \\
\hline Laparoscopic & $42(70.0)$ & $280(62.9)$ & \\
\hline Tumor size $(\mathrm{mm}$, mean $\pm \mathrm{SD})$ & $47.3 \pm 37.5$ & $37.5 \pm 23.6$ & 0.056 \\
\hline Tumor location, N. (\%) & & & 0.001 \\
\hline Renal pelvis & $33(55.0)$ & $152(34.2)$ & \\
\hline Ureter & $16(26.7)$ & $236(53.0)$ & \\
\hline Both & $11(18.3)$ & $57(12.8)$ & \\
\hline Previous or synchronous NMIBC, N. (\%) & & & 0.485 \\
\hline No & $46(76.7)$ & $362(81.3)$ & \\
\hline Yes & $14(23.3)$ & $83(18.7)$ & \\
\hline Multifocality, N. (\%) & & & 0.233 \\
\hline No & $37(61.7)$ & $312(70.1)$ & \\
\hline Yes & $23(38.3)$ & $133(29.9)$ & \\
\hline Pathologic T stage, N. (\%) & & & 0.026 \\
\hline Ta, CIS, T1-2 & $27(45.0)$ & $268(60.2)$ & \\
\hline T3-4 & $33(55.0)$ & $177(39.8)$ & \\
\hline Pathologic N. stage, N. (\%) & & & 0.748 \\
\hline $\mathrm{Nx}$ & $27(45.0)$ & $191(42.9)$ & \\
\hline No & $29(48.3)$ & $230(51.7)$ & \\
\hline N.+ & $4(6.7)$ & $24(5.4)$ & \\
\hline Grade, N. (\%) & & & 0.533 \\
\hline Low & $18(30.0)$ & $115(40.2)$ & \\
\hline High & $42(70.0)$ & $330(59.8)$ & \\
\hline Concomitant CIS, N. (\%) & & & 0.291 \\
\hline No & $58(96.7)$ & $401(92.2)$ & \\
\hline Yes & $2(3.3)$ & $34(7.8)$ & \\
\hline Lymphovascular invasion, N. (\%) & & & 0.110 \\
\hline No & $44(73.3)$ & $367(82.5)$ & \\
\hline Yes & $16(26.7)$ & $78(17.5)$ & \\
\hline Margin status, N. (\%) & & & 0.001 \\
\hline Negative & $54(90.0)$ & $425(95.5)$ & \\
\hline Positive & $6(10.0)$ & $20(4.5)$ & \\
\hline Adjuvant chemotherapy, N. (\%) & & & 0.493 \\
\hline No & $45(75.0)$ & $351(78.9)$ & \\
\hline Yes & $15(25.0)$ & $94(21.1)$ & \\
\hline
\end{tabular}

NMIBC: non-muscle-invasive bladder cancer; UTUC: upper tract urothelial carcinoma; CIS: carcinoma in situ; SD: standard deviation; BMI: Body Mass Index; ASA: American Society of Anesthesiologists.

BCE (log-rank test, $\mathrm{P}=0.024$ ) in the subgroup analysis of patients with ureteral cancer. When we performed subgroup analyses according to stage, there were no significant differences in intravesical recurrence-free survival, local/distant metastasis-free survival and CSS between 


\section{COPYRIGHT $^{(} 2017$ EDIZIONI MINERVA MEDICA}
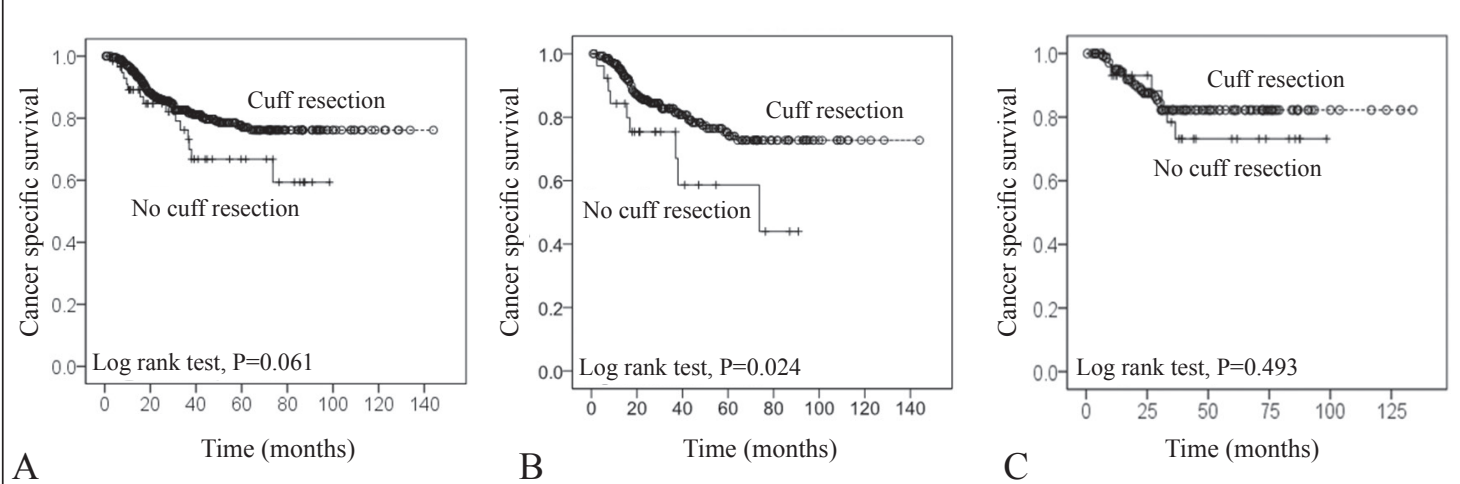

Figure 1.-Kaplan-Meier curves for cancer-specific survival according to bladder cuff management. A) All patients; B) ureteral cancer subgroup; C) renal pelvis tumor subgroup.

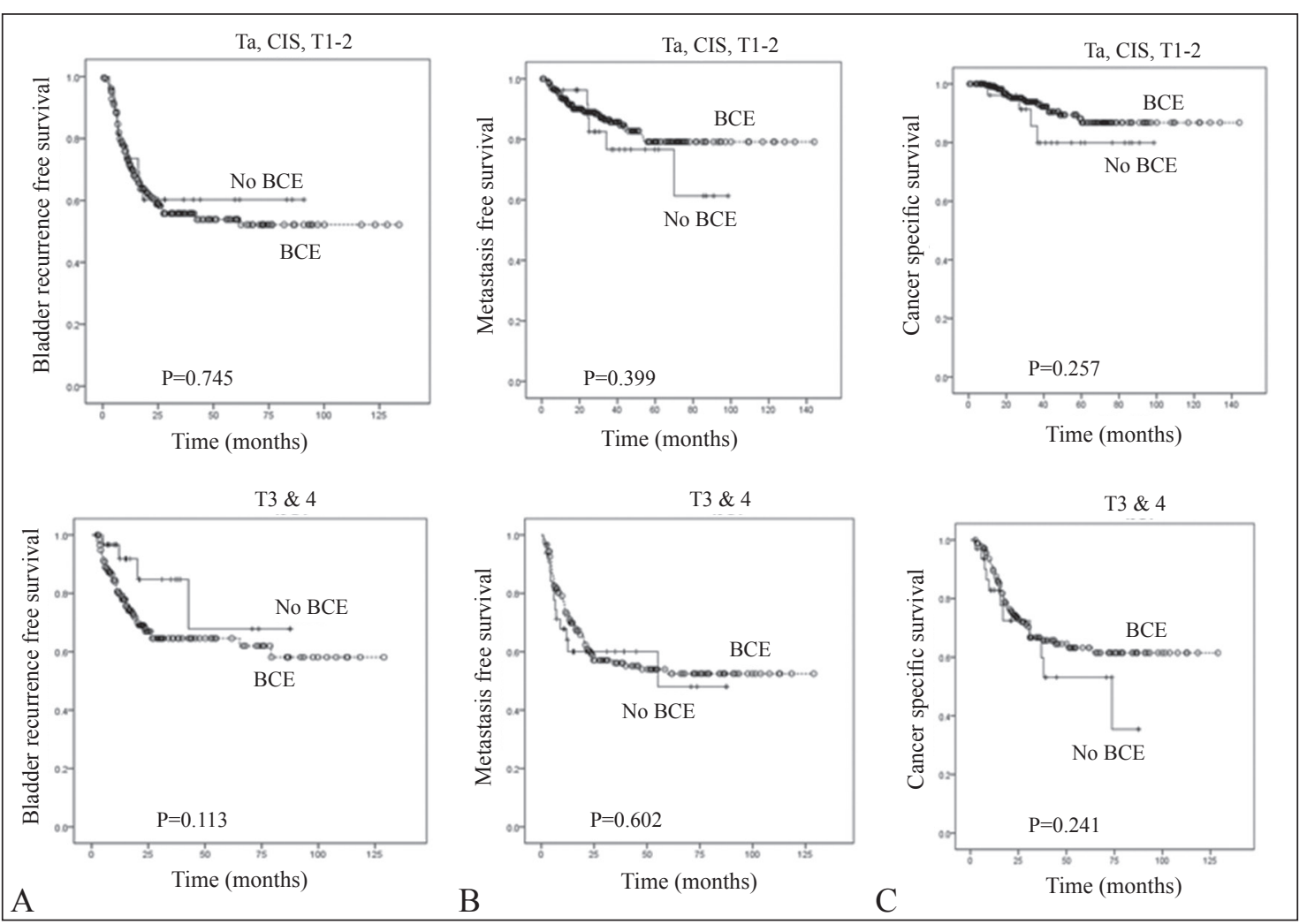

Figure 2.-Kaplan-Meier curves for intravesical recurrence-free survival (A), metastasis free survival (B) and cancer specific survival $(\mathrm{C})$ according to bladder cuff management stratifying by stage.

BCE: bladder cuff excision.

patients who underwent and did not undergo BCE stratifying by stage (Figure 2). In high grade tumors, bladder recurrence had a significant difference between patients with BCE and those without (Figure 3). Among the low grade tumors, patients without BCE had worse local/distant metastasis-free survival and CSS (Figure 3).

We further evaluated the clinicopathological characteristics of ureteral cancer in patients 


\section{COPYRIGHT ${ }^{\odot} 2017$ EDIZIONI MINERVA MEDICA}

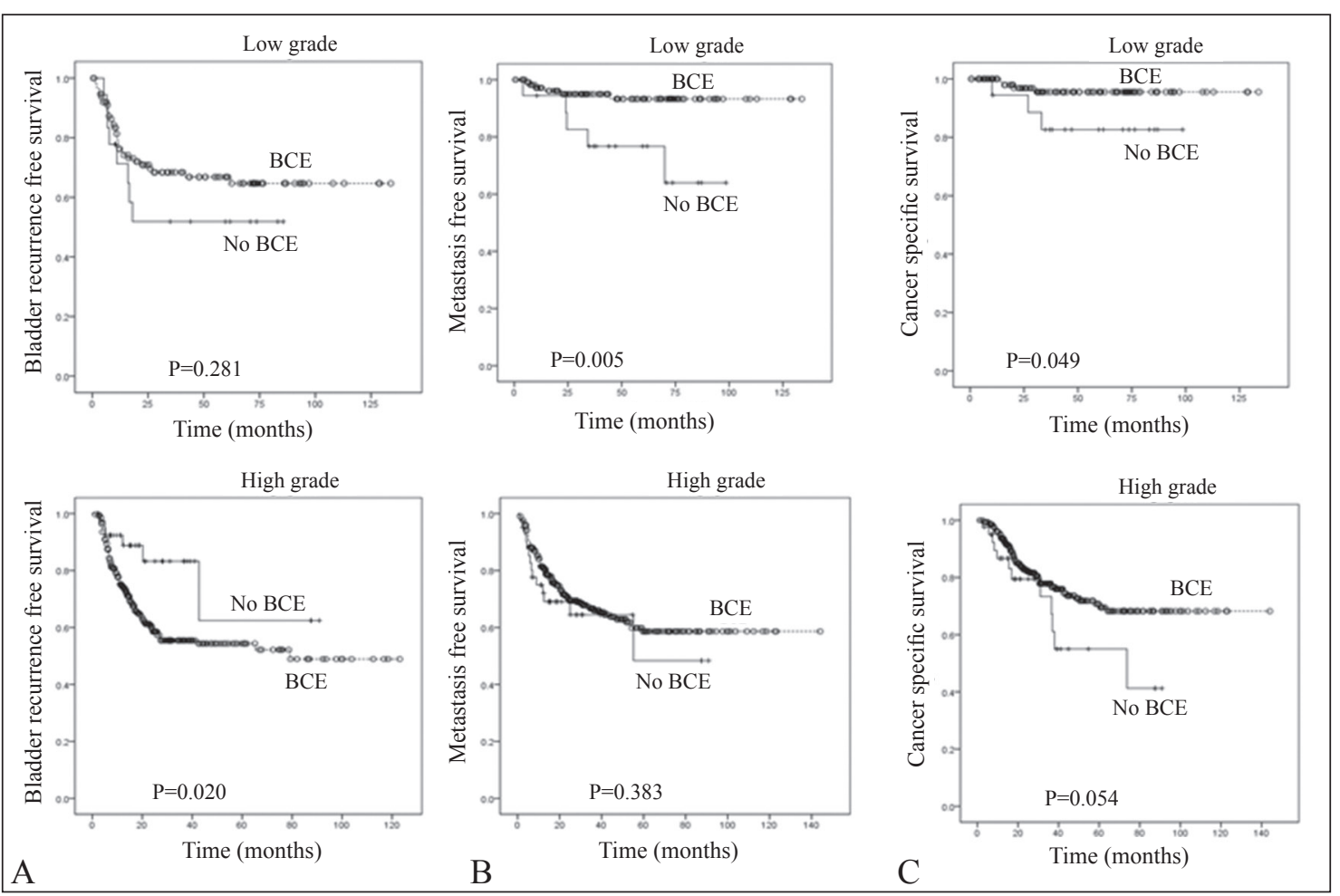

Figure 3.-Kaplan-Meier curves for intravesical recurrence-free survival (A), metastasis-free survival (B) and cancer-specific survival (C) according to bladder cuff management stratifying by grade.

BCE: bladder cuff excision.

who underwent RNU with and without BCE. Among the patients with ureteral cancer, there were no significant differences in the clinicopathological parameters except the surgical margin status $(\mathrm{P}=0.010)$ between the two groups (Table II). Multivariate analysis identified BCE (hazard ratio, 0.232 [95\% confidence interval, 0.075-0.717]; $\mathrm{P}=0.011$ ) as an independent prognostic factor of CSS in patients with ureteral cancer (Table III).

\section{Discussion}

UTUC cells can spread via the urine and seed in the underlying urothelium of the entire urinary tract. ${ }^{16}$ Because of these unique features, patients who undergo simple nephrectomy for UTUC have high recurrence rates of $33-70 \%$ in the remaining ureteral stump. ${ }^{17}$ RNU with BCE, including the intramural portion and the orifice of the ipsilateral ureter, is the current standard surgical approach for the treatment of UTUC. ${ }^{3}$ However, not all RNUs are performed with BCE. Moreover, there have not been any definitive conclusions concerning the prognostic value of $\mathrm{BCE}$ in the Korean UTUC patient cohort. In order to address the effect of $\mathrm{BCE}$ on the rate of cancer-specific mortality, we performed a multi-institutional study in patients with UTUC who were treated with RNU. Our observations revealed two important findings.

First, the patients who underwent RNU without BCE were older, had more comorbidities, and tumors with higher pathologic stage that were localized to the renal pelvis. Therefore, some of the patients were ineligible to undergo $\mathrm{BCE}$ owing to inevitable reasons. It is also noteworthy that although BCE improves survival, it may increase morbidity. For example, during open or laparoscopic operation, BCE may require a second incision. Furthermore, this procedure requires more time and wider dissection and increases the complexity of sur- 


\section{COPYRIGHT ${ }^{\odot} 2017$ EDIZIONI MINERVA MEDICA}

TABLE II.-Clinicopathological characteristics of ureteral cancer patients with and without bladder cuff excision.

\begin{tabular}{|c|c|c|c|}
\hline Parameters & $\begin{array}{l}\text { Patients without bladder } \\
\text { cuff excision }(\mathrm{N} .=27)\end{array}$ & $\begin{array}{l}\text { Patients with bladder } \\
\text { cuff excision }(\mathrm{N} .=293)\end{array}$ & $\mathrm{P}$ \\
\hline Age (years, mean \pm SD) & $72.0 \pm 8.7$ & $66.6 \pm 10.4$ & 0.067 \\
\hline BMI $\left(\mathrm{kg} / \mathrm{m}^{2}\right)$ & $23.9 \pm 2.4$ & $23.9 \pm 3.2$ & 0.968 \\
\hline Sex, N. (\%) & & & 0.523 \\
\hline Men & $16(59.3)$ & $197(67.2)$ & \\
\hline Women & $11(40.7)$ & $96(32.8)$ & \\
\hline Smoking status, N. (\%) & & & 1.000 \\
\hline No & $19(70.4)$ & $202(63.1)$ & \\
\hline Yes & $8(29.6)$ & $91(28.9)$ & \\
\hline ASA score, N. (\%) & & & 0.054 \\
\hline 1 & $2(7.4)$ & $71(24.2)$ & \\
\hline $2-4$ & $25(92.6)$ & $222(75.6)$ & \\
\hline Laterality, N. (\%) & & & 0.428 \\
\hline Left & $16(59.3)$ & $149(50.9)$ & \\
\hline Right & $11(40.7)$ & $144(49.1)$ & \\
\hline Operative method & & & 0.533 \\
\hline Open & $12(44.4)$ & $107(36.5)$ & \\
\hline Laparoscopic & $15(55.6)$ & $186(63.5)$ & \\
\hline Tumor size $(\mathrm{mm}$, mean $\pm \mathrm{SD})$ & $52.4 \pm 49.6$ & $35.6 \pm 22.2$ & 0.092 \\
\hline Previous or synchronous NMIBC, N. (\%) & & & 0.228 \\
\hline No & $18(66.7)$ & $229(78.2)$ & \\
\hline Yes & $9(33.3)$ & $64(21.8)$ & \\
\hline Multifocality, N. (\%) & & & 0.059 \\
\hline No & $13(48.1)$ & $196(66.9)$ & \\
\hline Yes & $14(51.9)$ & $97(33.1)$ & \\
\hline Pathologic T stage, N. (\%) & & & 0.095 \\
\hline Ta, CIS, T1-2 & $13(48.1)$ & $191(65.2)$ & \\
\hline T3-4 & $14(51.9)$ & $102(34.8)$ & \\
\hline Pathologic N stage, N. (\%) & & & 0.750 \\
\hline $\mathrm{Nx}$ & $10(37.0)$ & $128(43.7)$ & \\
\hline No & $15(55.6)$ & $150(51.2)$ & \\
\hline N. + & $2(7.4)$ & $15(5.1)$ & \\
\hline Grade, N. (\%) & & & 0.254 \\
\hline Low & $4(14.8)$ & $74(25.3)$ & \\
\hline High & $23(85.2)$ & $219(74.7)$ & \\
\hline Concomitant CIS, N. (\%) & & & 0.762 \\
\hline No & $25(92.6)$ & $265(90.4)$ & \\
\hline Yes & $2(7.4)$ & $28(9.6)$ & \\
\hline Lymphovascular invasion, N. (\%) & & & 0.290 \\
\hline No & $20(74.1)$ & $243(82.9)$ & \\
\hline Yes & $7(25.9)$ & $50(17.1)$ & \\
\hline Margin status, N. (\%) & & & 0.010 \\
\hline Negative & $21(77.8)$ & $275(93.9)$ & \\
\hline Positive & $6(22.2)$ & $18(6.1)$ & \\
\hline
\end{tabular}

NMIBC: non-muscle-invasive bladder cancer; UTUC: upper tract urothelial carcinoma; CIS: carcinoma in situ; SD: standard deviation; BMI: Body Mass Index; ASA: American Society of Anesthesiologists.

gery. Therefore, surgeons used to omit BCE, while performing a surgery for older adult patients or those having multiple comorbidities. However, to the best of our knowledge, the added morbidity has not been formally quantified.

Second, we confirmed the gain of survival benefit conferred by BCE only in patients with ureteral cancer. In general, renal pelvis tumor has a more favorable prognosis than ureter tumor. The reason is that ureteral wall is thin and easy to invade to adjacent area. Based on these results, BCE should be mandatory in these patients. Conversely, in patients with renal pelvic tumors, $\mathrm{BCE}$ omission does not undermine survival. Patients with incomplete nephro- 


\section{COPYRIGHT $^{\odot} 2017$ EDIZIONI MINERVA MEDICA}

BLADDER CUFF EXCISION DURING RADICAL NEPHROURETERECTOMY

HA

TABLE III.-Multivariate Cox regression analysis of the predictors of cancer related death in ureteral cancer.

\begin{tabular}{lrrrr}
\hline & & \multicolumn{2}{c}{$95 \%$ CI } & P \\
\cline { 3 - 4 } Parameters & & Lower & Upper & \\
\hline Age & 1.124 & 1.051 & 1.203 & 0.001 \\
Sex (men vs. women) & 1.114 & 0.370 & 3.357 & 0.848 \\
Smoking (no vs. yes) & 1.046 & 0.331 & 3.302 & 0.939 \\
ASA score (1 vs. 2-4) & 0.692 & 0.234 & 2.052 & 0.507 \\
Pathologic T stage (Ta, CIS, T1-2 vs. T3-4) & 3.711 & 1.116 & 12.337 & 0.032 \\
Pathologic N stage (Nx, N0 vs. N+) & 15.673 & 4.621 & 53.159 & $<0.001$ \\
Grade (low vs. high) & 2.053 & 0.228 & 18.466 & 0.521 \\
Lymphovascular invasion (no vs. yes) & 2.080 & 0.654 & 6.613 & 0.215 \\
Multifocality (no vs. yes) & 2.161 & 0.802 & 5.826 & 0.128 \\
Bladder cuff excision (no vs. yes) & 0.232 & 0.075 & 0.717 & 0.011 \\
Margin status (no vs. yes) & 0.914 & 0.259 & 3.223 & 0.888 \\
Previous or synchronous NMIBC (no vs. yes) & 1.141 & 0.410 & 3.174 & 0.800 \\
Concomitant CIS (no vs. yes) & 1.982 & 0.392 & 10.033 & 0.408 \\
\hline NIBC: no & & &
\end{tabular}

NMIBC: non-muscle-invasive bladder cancer; UTUC: upper tract urothelial carcinoma; CIS: carcinoma in situ; BMI: Body Mass Index; ASA: American Society of Anesthesiologists; HR: hazard ratio; CI: confidence interval.

ureterectomy are at increased risk for bladder cancer and potentially decreased survival; 18 however, BCE did not affect bladder recurrence in the present series. Kang et al. showed that patients who did not undergo BCE had poorer overall survival and CSS after RNU than those who underwent BCE. ${ }^{19}$ In a Korean study by Kim et al., bladder cuffing was the sole independent prognostic factor for disease specific survival among patients with stage III or IV UTUC..$^{20}$ Lughezzani et al. evaluated the prognostic significance of BCE during RNU for CSS in a large-scale population-based cohort of patients with renal pelvic tumors. ${ }^{12}$ They showed that there was no increase in cancer-related death in patients with localized $(\mathrm{pT} 1-2 \mathrm{~N} 0 / \mathrm{x})$ renal pelvic tumors when $\mathrm{BCE}$ was omitted and that CSS might decrease significantly if BCE was not performed in patients with locally advanced (pT3-4N0/x and $\mathrm{pT}[\mathrm{any}] \mathrm{N} 1-3)$ renal pelvic tumors. In the present study, there were no significant differences in intravesical recurrence-free survival, local/ distant metastasis-free survival and CSS between patients who underwent and did not undergo BCE stratifying by stage. Although we did not know exact reasons, ethnic differences may affect these results. This information may help patient categorization and indicate RNU without BCE in specific patients. The results of the present study provided useful information for the management of UTUC patients, particularly ureteral cancer. In subgroups of ureteral cancer, BCE had a significant impact on CSS. Therefore, BCE should invariably be performed in patients with ureteral cancer.

\section{Limitations of the study}

The present study had several limitations. First, the comparison was not balanced. RNU with BCE is a standard operation for UTUC. When we had not performed BCE, there were several inevitable reasons although it is against oncological principles. Therefore the study population of this group cannot be large. Second, it is limited by its retrospective nature and missing data as well as the heterogeneity of the patient population, variations in the follow-up schedule of each institution, and short median follow-up duration. However, when the data were reanalyzed by including the information on the institution, the statistical significance of the variables did not change, and the institutional differences did not affect disease recurrence or mortality following RNU. Third, unfortunately, our database did not contain detailed ureteral cuff management. So we did not know exactly how much of the ureteric stump was left. Lastly, the absence of data on ureteral stump recurrences prevented us from concluding that RNU without BCE should represent a standard of care for all patients with renal pelvic tumors. Most patients who did not un- 


\section{COPYRIGHT ${ }^{\odot} 2017$ EDIZIONI MINERVA MEDICA}

dergo BCE experienced tumor recurrence at the remnant ureter. ${ }^{19}$ In a study by Kang et al., $40.0 \%$ of the patients who underwent BCE experienced ureteral stump recurrence, which aggravated the patient prognosis. ${ }^{19}$ In the present study, there were no significant differences in intravesical recurrence-free survival and local/distant metastasis-free survival between patients who underwent and did not undergo $\mathrm{BCE}$ even in subgroup analyses of renal pelvic tumors and ureteral cancer. Radical surgery and adjuvant chemotherapy might give the therapeutic effects on these results.

\section{Conclusions}

Despite its limitations, the present study demonstrated that CSS might decrease significantly in patients with ureteral cancer who did not undergo BCE. Therefore, BCE should become the standard of care during RNU in these patients. Conversely, no decrease in the CSS was recorded in patients with renal pelvic tumors when BCE was omitted from the treatment. However, BCE should only be omitted in specific cases for which this option is inevitable.

\section{References}

1. Raman JD, Messer J, Sielatycki JA, Hollenbeak CS. Incidence and survival of patients with carcinoma of the ureter and renal pelvis in the USA, 1973-2005. BJU Int 2011;107:1059-64.

2. Siegel RL, Miller KD, Jemal A. Cancer statistics, 2015. CA Cancer J Clin 2015;65:5-29.

3. Roupret M, Babjuk M, Comperat E, Zigeuner R, Sylvester R, Burger M, et al. European Association of Urology Guidelines on Upper Urinary Tract Urothelial Cell Carcinoma: 2015 Update. Eur Urol 2015;68:868-79.

4. Mathieu R, Bensalah K, Lucca I, Mbeutcha A, Roupret M, Shariat SF. Upper urinary tract disease: what we know today and unmet needs. Transl Androl Urol 2015;4:26172.

5. Lee JN, Kim BS, Kim HT, Kim TH, Yoo ES, Choi GS, et al. Oncologic outcomes of laparoscopic nephroureterectomy for $\mathrm{pT} 3$ upper urinary tract urothelial carcinoma. Minerva Urol Nefrol 2014;66:157-64.
6. Kang HW, Jung HD, Ha YS, Kim TH, Kwon TG, Byun SS, et al. Preoperative underweight patients with upper tract urothelial carcinoma survive less after radical nephroureterectomy. J Korean Med Sci 2015;30:1483-9.

7. Kim BW, Ha YS, Lee JN, Kim HT, Kim TH, Lee JK, et al. Effects of previous or synchronous non-muscle invasive bladder cancer on clinical results after radical nephroureterectomy for upper tract urothelial carcinoma: a multi-institutional study. Urol J 2015;12:2233-9.

8. Capitanio U, Shariat SF, Isbarn H, Weizer A, Remzi M, Roscigno $\mathrm{M}$, et al. Comparison of oncologic outcomes for open and laparoscopic nephroureterectomy:a multiinstitutional analysis of 1249 cases. Eur Urol 2009;56:19.

9. Srirangam SJ, van Cleynenbreugel B, van Poppel H. Laparoscopic nephroureterectomy: the distal ureteral dilemma. Adv Urol 2009:316807.

10. Abel EJ, Fisher MB, Matin SF, Kamat AM, Dinney CP, Grossman HB. Delayed ureterectomy after incomplete nephroureterectomy for upper tract urothelial carcinoma: pathologic findings and outcomes. Int Braz J Urol 2013;39:817-22.

11. Zlotta AR. Should urologists always perform a bladder cuff resection during nephroureterectomy, and which method should they use? Eur Urol 2010;57:970-2.

12. Lughezzani G, Sun M, Perrotte P, Shariat SF, Jeldres C, Budaus L, et al. Should bladder cuff excision remain the standard of care at nephroureterectomy in patients with urothelial carcinoma of the renal pelvis? A populationbased study. Eur Urol 2010;57:956-62.

13. Lughezzani G, Jeldres C, Isbarn H, Sun M, Shariat SF, Alasker A, et al. Nephroureterectomy and segmental ureterectomy in the treatment of invasive upper tract urothelial carcinoma:a population-based study of 2299 patients. Eur J Cancer 2009;45:3291-7.

14. Greene FL. The American Joint Committee on Cancer: updating the strategies in cancer staging. Bull Am Coll Surg 2002;87:13-5.

15. Epstein JI, Amin MB, Reuter VR, Mostofi FK. The World Health Organization/International Society of Urological Pathology consensus classification of urothelial (transitional cell) neoplasms of the urinary bladder. Bladder Consensus Conference Committee. Am J Surg Pathol 1998;22:1435-48.

16. Booth CM, Cameron KM, Pugh RC. Urothelial carcinoma of the kidney and ureter. Br J Urol 1980;52:430-5.

17. McCarron JP, Mills C, Vaughn ED Jr. Tumors of the renal pelvis and ureter: current concepts and management. Semin Urol 1983;1:75-81.

18. Hou CP, Chang PL, Chen CL, Lin YH, Tsui KH. Does adequate bladder cuff excision impact outcomes in patients undergoing nephroureterectomy for upper tract urothelial carcinoma. Chang Gung Med J 2011;34:496-505.

19. Kang M, Jeong CW, Kwak C, Kim HH, Ku JH. The characteristics of recurrent upper tract urothelial carcinoma after radical nephroureterectomy without bladder cuff excision. Yonsei Med J 2015;56:375-81.

20. Kim HS, Lee JS, Jeong CW, Kwak C, Kim HH, Ku JH Adjuvant chemotherapy for locally advanced upper tract urothelial carcinoma: updated results of the Seoul National University Hospital experience. Int Braz J Urol 2015;41:1067-79.

Funding.-This work was supported by the National Research Foundation of Korea (NRF) grant funded by the Korean government (MSIP) (No. 2016R1C1B1011180).

Conflicts of interest.-The authors certify that there is no conflict of interest with any financial organization regarding the material discussed in the manuscript.

Article first published online: February 14, 2017. - Manuscript accepted: February 6, 2017. - Manuscript revised: January 30, 2017. Manuscript received: September 13, 2016. 